Ann. Sci. For., 1985, 42 (1), 1-22

\title{
Contribution à l'étude du dépérissement du chêne : pathologie racinaire en forêt de Tronçais
}

\author{
J.J. GUILLAUMIN *, Ch. BERNARD*, C. DELATOUR ** \\ et Micheline BELGRAND $* *$ \\ *I.N.R.A., Station de Pathologie végétale, \\ 12, awenue du Brézet, F 63039 Clemont-Ferrand \\ ** I.N.R.A., Laboratoire de Pathologie forestiere,
}

Centre de Recherches forestières de Nancy, Champenoux, F 54280 Seichanps

**** I.N.R.A., Station de Recherches sur les Sols forestiers et la Fertilisation

Conire de Recherches forestiores de Nancy, Champenoux, f 54280 Seichempins

\section{Résumé}

Le rôle joué par les Basidionycètes parasites des racines dans le dépérissement du chêne pédonculé a été étudié en forêt de Tronçais (Allier).

L'initjation du dépérissement est d'origine édapho-climatique : la sécheresse de 1976 a joué le rôle inducteur, mais seuls les arbres situés dans des stations difficiles (nappe phréatique ou niveau d'induration proche de la surface) ont dépéri.

Le rôle des champignons des racines a été étudié grâce à diverses techniques d'investigation, notamment le dégagement complet de systèmes racinaires à la pelle mécanique. l'étude de l'évolution individuelle de chaque arbre depuis 1975 a été effectuée par des méthodes dendrochronologiques.

Il est apparu que les Basidiomycètes des racines jouent un rôle non négligeable : si l'on ne peut pas affirmer qu'ils interviennent dans le début du phénomène de dépérissement, leur présence ou leur absence peut expliquer que certains arbres dépérissants soient morts tandis que d'autres se rétablissaient à partir de 1979-1980. Trois espèces de Basidiomycètes jouent, dans ce processus, le rôle essentiel : Qıél.

- d'une part, les Armillaires A. mellea et $A$. bulbosa;

- d'autre part, une Agaricale, déterminée comme étant Collybia fusipes (Bull. ex Fr.)

- Les Armillaires prédominent en sols hydromorphes. A. mellea semble intervenir plus précocement que $A$. bulbosta, clle affecte des racines de plus petit diamètre et situées plus loin de la souche.

- La Collybie prédomine nettement en sols non hydromorphes. Cctle espèce est fréquente sur les arbres sains (y compris les chênes sessiles) sous forme de lésions latcntes circonscrites par des couches de liège. Sur les arbres victimes de stress écologiques, ees lésions deviennent évolutives et l'on observe une pourriture orangée de l'écorce et de l'aubier qui peut se généraliser à l'ensemble du système racinaire. 


\section{Introduction}

Depuis 1978, un dépérissement des chênes de 70 à 120 ans s'est développé en France, essentiellement dans 2 régions : les plaines de la France centrale, du Berry au Val-de-Saône, et les côteaux sous-pyrénéens (Pays Basque et Béarn) (BuFFET, 1983). Ce phénomène rappelait par certains de ses caractères d'autres dépérissements du chêne survenus en France, notamment pendant les périodes 1921-1926 et 1942-1948. Il a fait l'objet d'une étude de la part d'un groupe interdiciplinaire dont l'activité s'est centrée surtout sur la forêt de Tronçais (Allier).

Il est apparu clairement qu'en forêt de Tronçais, le dépérissement des chênes ne s'est pas développé au hasard. BECKER \& LeVY (1982) ont en effet mis en évidence d'une part que seul le Pédonculé $(Q$. robur. L.) en était la victime et d'autre part que l'intensité du phénomène variait grandement sclon le type de station. En outre, ces mêmes auteurs après examen des accroissements radiaux des années récentes ont confirmé l'hypothèse de l'affaiblissement engendré par la sécheresse de 1976, le bon rétablissement du Sessile $(Q$. petraea), le rétablissement variable du Pédonculé. L'ampleur de l'accident climatique a été analysée par l'un de nous (BERNARD. 1982) ; la sécheresse de 1976 a non seulement coincidé avec la période de végétation active mais a aussi succédé à une période hivernale particulièrement déficitaire en précipitations ; il faut remonter aux années 1944-1949 pour observer des déficits pluviométriques comparables.

L'accident climatique qui précède le dépérissement de certains chênes est un fait souvent cité dans la littérature (Delatour, 1983) ainsi que d'autres facteurs d'affaiblissement tels que les insectes défoliateurs et l'Ö̈dium. A Tronçais, la Tordeuse verte aurait sévi en 1977-1978 et l'Oiddium en 1977, majs leur importance demeure difficile à apprécier a posteriori. Elle est vraisemblablement faible.

Enfin, comme dans beaucoup de cas antérieurement étudićs, l'Armillairc était fréquemment observée sur les arbres dépérissants ou morts. Il apparaît donc que ce champignon, composant normal de la flore mycologique des chênaies, modifie son comportement et devient parasite lorsque les arbres sont affaiblis. On peut alors supposer qu'en liaison avec le dépérissement, d’importantes perturbations se produisent dans les systèmes racinaires et le rôle de l'Armillaire à cet égard mérite d'être précisé ; on peut en outre considérer conme improbable que l'Armillaire soit seule à intervenir à ce niveau compte tenu de la complexité du biotope forestier.

L'objectif de notre travail a été de préciser la nature des perturbations racinaires, en insistant sur l'aspect mycologique, et d'en apprécier la portée. Afin que nos résultats puissent prendre toute leur signification écologique, il est apparu indispensable d'une part de réaliser nos observations dans des conditions stationnelles parfaitement définies et d'autre part de rendre la plus objective possible l'appréciation de l'état de dépérissement des arbres étudiés.

Nous avons effectué cette étude en utilisant 2 méthodes :

1) Sur un grand nombre de chênes (197 au total), les observations se sont limitées à la partie superficielle du système racinaire : le collet ct le clépart des grosses racines. 
2) Sur un petit nombre d'arbres, il a pu être procédé au dégagement d'une partie importante du système racinaire, ce qui a permis d'effectuer une étude de la morphologie et de la structure des systèmes racinaires (dont les résultats figurent dans la 2 " partic de cet article) el également une étude pathologique (3" partie de l'article).

Ces 2 méthodes nous ont semblé complémentaires; la première permet d'observer un nombre d'arbres suffisant pour permettre des comparaisons statistiques, mais il est hasardeux de déduire l'état de la totalité du système racinaire d'une simple observation des parties superficielles. La seconde méthode donne accès à l'ensemble du système racinaire, mais pour des raisons matérielles évidentes, elle ne peut porter que sur quelques arbres.

\section{Les bases de l'étude}

Les observations ont été réalisées dans des placettes définies selon leurs caractéristiques stationnclles et leur degré de dépérissement.

BECKER \& Levy ayant montré l'existence en forêt de Tronçais de 4 types distincts de stations, nous avons été amenés à opérer certains rapprochements selon que les sols de ces stations étaient ou non hydromorphes.

L'appréciation du dépérissement au niveau de la placette a été basée sur les données recueillies par photographie aérienne (Rıом, 1981). Les placettes à «faible dépérissement " on été établies dans des zones bien pourvues en arbres dont la réflectance IR s'éloignait de celle du peuplement sain environnant (arbres " affaiblis " sans descente de cime), les mortalités y étaient rares. Les placettes à "fort dépérissment » ont été choisies dans des zones où les descentes de cime et les mortalités étaient abondantes.

Selon ces critères, 4 types de placettes ont été définis (tabl. 1).

A lintérieur de chaque placette, un certain nombre de chênes ont été désignés (au total 197) en distinguant l'espèce botanique et le degré de dépérissement. A ce stade, 4 catégories ont été appréciées par examen visuel des houppiers :

1) Chênes sains : houppier sans symptômes.

2) Chênes peu dépérissants : cime légèrement dégarnie, feuilles jaunies ça et là, aspect général peu vigoureux.

3) Chênes très dépérissants : plusieurs branches mortes (parfois descente de cime), feuillage souvent rare et disposé en manchon sur quelques grosses branches.

4) Chênes morts.

Ces arbres ont ensuite, pour la plupart d'entre eux, fait l'objet d'un sondage à la tarière à 1,30 $\mathrm{m}$ et les accroissements des 15 dernières années ont été étudiés sur radiographie.

Conformément à ce qui a été indiqué par ailleurs par BECKER et Levy (1982) il est possible de classer les chênes en 2 grandes catégories selon que la croissance radiale est ou non en augmentation après les années 1976-1977 (les quelques cas d'accroissement réellement stationnaires ont pu être répartis dans les 2 catégories précédentes selon une norme précise (BERNARD, 1982). 


\section{TABIEAU 1}

Description des parcelles étudiées.

Description of the plots examined.

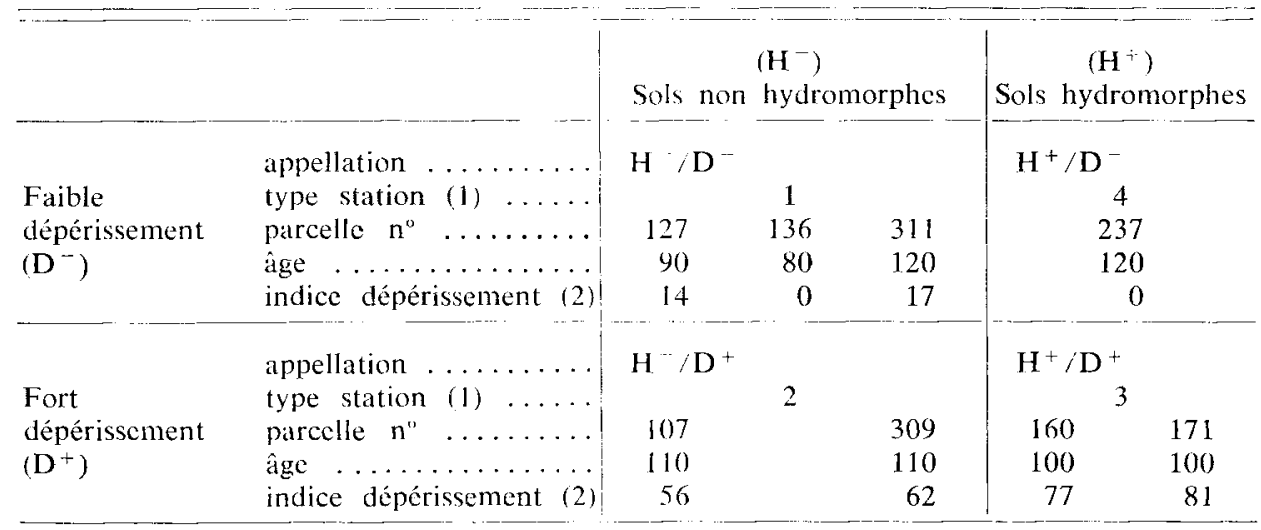

(1) BECKER, LEVY (1982).

(2) $\frac{\text { nombre arbres morts }}{\text { nombre arbres morts }+ \text { aflaiblis }}$ (RION, 1981).

La comparaison entre les 2 méthodes d'appréciation du dépérissement est résumée dans le tableau ci-dessous pout les chênes pédonculés (136 arbres) :

\section{TABLEAU 2}

Appréciation du degré de dépérissement chez 136 chênes pédonculés.

Comparaison entre l'appréciation visuelle et la croissance radiale.

Percent of trees which recovered and did not recovered from dieback, determined by radial growth patterns compared with estimates of tree vigor determined visually (136 trees evaluated).

\begin{tabular}{|c|c|c|c|}
\hline & & \multicolumn{2}{|c|}{ Croissance radiale } \\
\hline & & reprise & non reprise \\
\hline \multirow{3}{*}{ Appréciation visuelle } & Sains $\ldots \ldots \ldots$ & $88 \%$ & $12 \%$ \\
\hline & Peu dépérissants $\ldots \ldots \ldots \ldots$ & $63 \%$ & $37 \%$ \\
\hline & Très dépérissants $\ldots . .$. & $8 \%$ & $92 \%$ \\
\hline
\end{tabular}


L’appréciation visuelle des arbres «sains» comme des «très dépérissants » concorde bien avec ce qui est observé au niveau des accroissements radiaux (concordance à 88 p. 100 et 92 p. 100 respectivement). Nous voyons par contre que parmi les arbres considérés comme «peu dépérissants » une proportion importante (37 p. 100) ne présente aucune reprise des accroissements radiaux.

L'accroissement radial intègre de nombreux facteurs et permet de quantificr un certain état de vigueur des arbres et surtout son évolution. Il est donc certainement plus fiable et plus objectif de juger l'état de dépérissement des arbres d'après ce critère ; c'est donc sur lui seul que nous avons par la suite basé l'appréciation du dépérissement. Nous n'avons alors plus considéré que 2 catégories à l'intérieur des chênes pédonculés : les arbres rétablis et les arbres non rétablis. On ne peut pas tirer de conclusions sylvicoles précises de notre étude des accroissements car les arbres n'ont pas été choisis réellement au hasard. Nos résultats, résumés dans la fig. 1, confirment ecpendant ceux de Becker \& Levy (1982) : les chênes sessiles ont tous manifesté un bon rétablissement, en moyenne dès 1978 ; chez les pédonculés le rétablissement est plus tardif (1979). Il faut noter en outre que le groupe des pédonculés non rétablis présentait, en stations non hydromorphes, un accroissement moyen annuel avant 1975, toujours nettement inféricur à celui du groupe des pédonculés rétablis, ce phénomène ne se manifeste pas en stations hydromorphes.
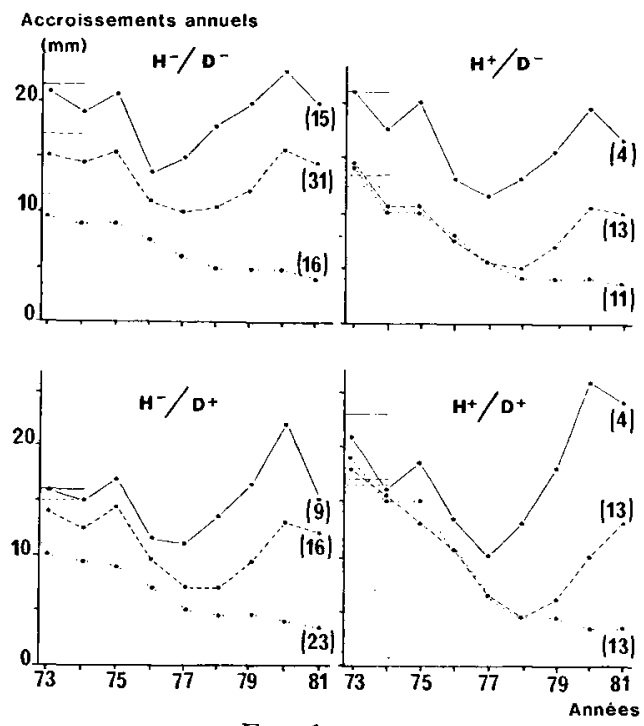

FIG. 1

Accroissement radiaux des chênes dans les quatre types de placettes étudiées.

Radial growth of oaks in the four types of plot studied.

Ligne pleine : $Q$. petraea.

Ligne tiretéc $: Q$, robur rétablis.

Ligne pointillée : $Q$. robur non rétablis.

Le trait horizontal à gauche de chaque courbe représente l'accroissement moyen annuel entre 1966 ct 1975 . Entre parenthèses : nombre d'arbres étudiés.

Solid line : $\mathrm{Q}$. petraca.

Dashed line : recovered $\mathrm{Q}$. robur.

Dotted line : not recovered $\mathrm{Q}$. robur.

The horizontal line at the left of each curve is the mean annual growth from 1966 to 1975. Values in brackets indicate the number of trees studied. 
La réduction des accroissements radiaux apparaît être dans tous les cas une conséquence de la sécheresse de 1976, mais le non rétablissement ultérieur de la croissance, c'est-à-dire le dépérissement proprement dit, semble d'interprétation plus délicate. $\mathrm{Il}$ apparaît intéressant de constater que dans certaines stations, les arbres ayant dépéri semblent avoir été prédisposés du fait d'une moindre vigueur. En effet, du point de vue de la pathologie racinaire où nous nous sommes plus particulièrement placés, cette constatation conduit à certaines hypothèses. En stations hydromorphes, l'intervention de pathogènes intcrdisant le rétablissement de certains arbres pourrait n'avoir été que consécutive à l'année 1976. Par contre, en stations non hydromorphes, elle pourrait avoir été antéricure, contribuant à la moindre vigueur des arbres qui ont cnsuite dépéri.

\section{Caractéristiques de l'enracinement}

Le dépérissement du chêne pédonculé à Tronçais apparaît être la conséquence de la sécheresse de 1976 sur des sols déjà défavorables à cette cspèce par leur régime hydrique. Ces derniers sont caractérisés entre autres par un faible volume de sol colonisable par les racines, car limité par l'existence d'un horizon induré peu profond ou par une nappe superficielle (BI:CKER \& LEVY, 1982).

11 semble d'après ces auteurs que la différence de réactions à la sécheresse de 1976 entre le pédonculé et le sessile puisse s'expliquer par leur écophysiologie et notamment leur comportement hydrique; à ce niveau le type d'enracinement peut joucr un rôle dans les potentialités d'alimentation en cau des deux essences.

Les objectifs de cette partic de notre étude étaient d'une part de vérifier si une différenciation de la morphologie racinaire ne pouvait pas expliquer la différence de comportements et d'autre part d'observer l'importance de la nature des atlaques parasitaires sur une fraction importante du système racinaire (voir la 3" partie).

\subsection{Méthodologie}

L'étude a été réalisée dans les placettes de type $\mathrm{H}^{-} \mathrm{D}+$ (parcelle 107) el $\mathrm{H}^{+} \mathrm{D}+$ (parcelle 160).

Elle a porté à chaque fois sur un triplet d'arbres :

- chêne sessile,

- chêne pédonculé rétabli,

- chêne pédonculé non rétabli.

La lourdeur de ce type d'investigations n'a permis d'observer que 2 répétitions par station et espèce soit 6 arbres par station et 12 au total.

Les chênes implantés sur la station non hydromorphe $\left(\mathrm{H}^{-} \mathrm{D}^{-+}\right)$étaient âgés de 100 à 120 ans avec des diamètres compris entre 35 et $45 \mathrm{~cm}$ à $1,30 \mathrm{~m}$ de hautcur. Ceux situés dans la station à hydromorphie superficielle avaient le même âge mais leurs diamètres étaient légèrement inférieurs.

La méthode d'investigation utilisée est illustrée par la figure 2 : grâce à l'emploi d'une pelle mécanique, on a creusé deux fosses diamétralement opposées, de part 
et d'autre de chaque arbre à étudier. La pronfondeur de ces fosses était de 1,30 $\mathrm{m}$, leur distance à la souche de $2 \mathrm{~m}$ à $2,50 \mathrm{~m}$, leur longueur totale d'environ $6 \mathrm{~m}$ à $2 \mathrm{~m}$ de la souche. A partir de ces fosses, on a procédé au dégagement manuel du système racinaire, en progressant en direction de la souche. Toutes les racines entre 2 ares successifs de $0,50 \mathrm{~m}$ ont été prélevées par tranches de $10 \mathrm{~cm}$ de profondcur.

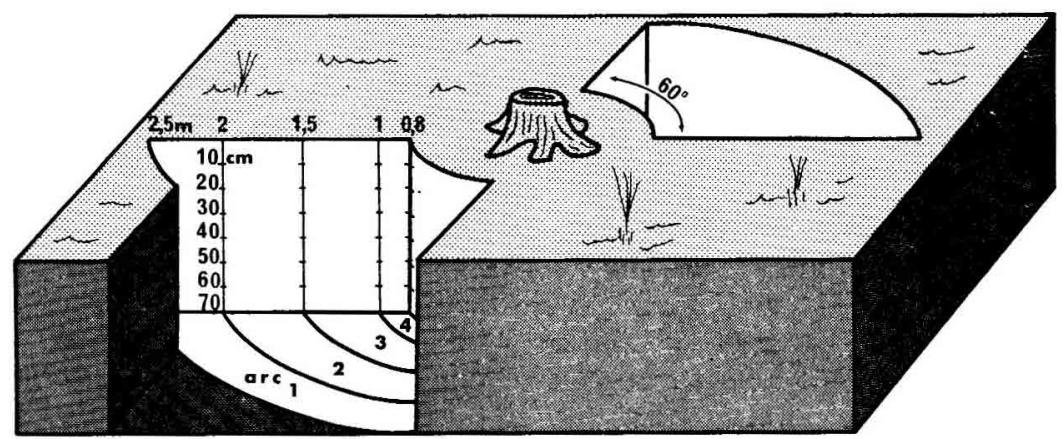

FIG. 2

Dispositif pour l'observation et le prélèvement des racines.

Type of excavations made for observation of roots and collection of samples.

On a ainsi pu observer plus de la moitié du système racinaire de chaque arbre. Les prélèvements ont été arrêtés à $0,80 \mathrm{~m}$ du centre de la souche. Cette dernière a alors été dégagée et photographiée, des échantillons ont été prélevés pour les examens pathologiques.

Les racines ont été rapportées au laboratoire et réparties en 4 classes de diamètre :
a - $\varnothing<5 \mathrm{~mm}$
b - $5 \leq \varnothing<20 \mathrm{~mm}$
c $-20 \leq \varnothing<50 \mathrm{~mm}$
d - $\varnothing>50 \mathrm{~mm}$

Chaque prélèvement était classé selon 3 critères (classe de diamètre, distance à la souche, profondeur), puis séché à l'étuve à 65 "C et pesé (biomasse en poids sec).

Les examens pathologiques étaient réalisés en parallèle.

\subsection{Résultats}

Le faible nombre de chênes examinés limite les conclusions qu'on peut tirer de cette étude; on peut cependant effectuer les remarques suivantes :

\subsubsection{Morphologie générale}

Dans la station non hydromorphe, on a constaté que les racines se trouvaicnt surtout dans un rayon de $2,50 \mathrm{~m}$ à partir de la souche; des observations, effectuées à une distance plus grande $(3,50 \mathrm{~m})$ ont montré la très faible densité racinaire au-delà de $2,50 \mathrm{~m}$. 
L'architecture racinaire des chênes comporte globalement plusieurs pivots ou un seul qui se ramifie au contact de lhorizon induré (entre 50 et $70 \mathrm{~cm}$ de profondeur). La progression des grosses racines plongeantes est rapidement stoppéc par l'induration du sol. Il existe aussi quelques racines subhorizontales de grand diamètre mais leur longueur et lcur nombre sont limités.

\subsubsection{Biomasse racinaire}

La figure 3 montre qu'en station non hydromorphe, les biomasses racinaires des chênes sessiles et pédonculés rétablis sont assez comparables. En revanche, le pédonculé non rétabli est caractérisé par une biomasse racinaire plus faible, surtout pour les racines moyennes $(5-50 \mathrm{~mm})$. Si l'on veut étudier le système racinaire en tant que facteur influençant les possibilités d'alimentation en eau des arbres, il est nécessaire d'examiner surtout les racines de faible diamètre.

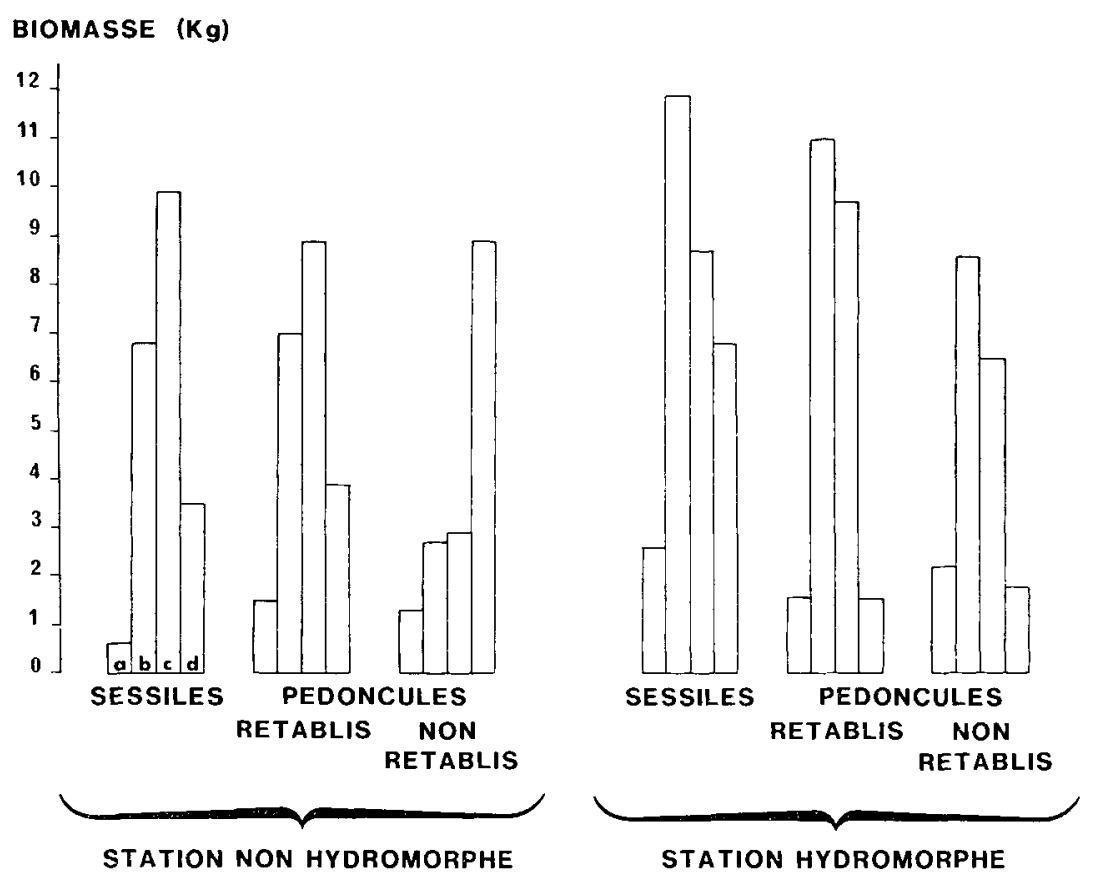

FIG. 3

Biomasses racinaires totales par classes de diametre (exprimées en $\mathrm{kg}$ de poids sec). Total root hiomass ( $\mathrm{kg}$ dry weight) by root diameter class.

a : racines de diamètre 0 à $5 \mathrm{~mm}$

b : racines de diamètre 5 à $20 \mathrm{~mm}$

c : racines de diamètre 20 à $50 \mathrm{~mm}$

d : racines de diamètre supérieur à $50 \mathrm{~mm}$

a: root diameter $0-5 \mathrm{~mm}$

b): root diameter $5.20 \mathrm{~mm}$

c: root diameter $20-50 \mathrm{~mm}$

$d$ : root diameter greater than $50 \mathrm{~mm}$ 
L'examen de la répartition en profondeur de la biomasse des racines de diamètre compris entre 0 et $50 \mathrm{~mm}$ montre que linduration limite très fortement la progression des racines en profondeur (figures 4 et 5 ) :

- si l'on cxaminc la répartition des très fines racines $(0-5 \mathrm{~mm})$ on observe de nettes différences entre espèces (fig. 4) : le sessile présente un «chevelu » particulièrement bien développé $s$ profondeur par rapport au pédonculé rétabli. Le pédonculé non rétabli est caractérisé par une quasi-absence de très fines racines en profondeur. Pour cette classe de diamètre, il semble donc que les sessiles prospectent plus profondément le sol que les pédonculés rétablis; ceci pourrait contribuer à une meilleure alimentation en eau des arbres;

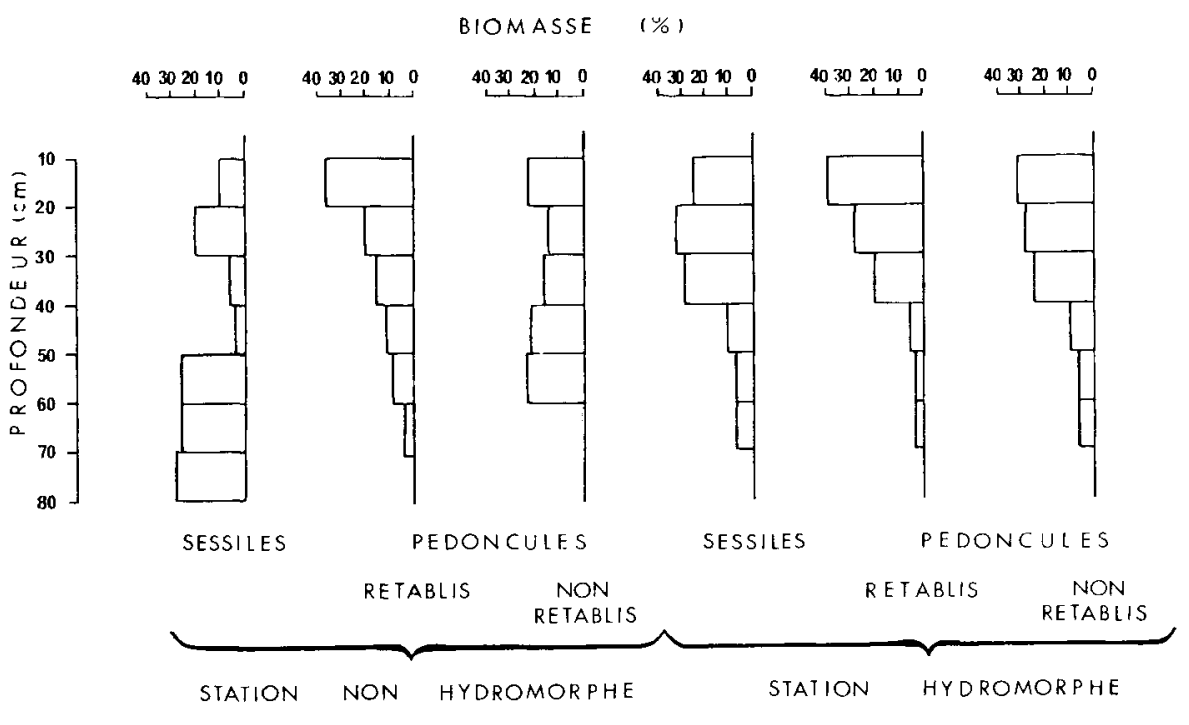

FIG. 4

Répartition selon la profondeur de la biomasse de très fines racines $(0-5 \mathrm{~mm})$ (cxprimée en p. 100 de la biomasse sèche totale des très fines racines).

Biomass of very fine roots (diam. 0-5 $\mathrm{mm}$ ) at different soil depths (expressed as p. 100 of total dry biomass of very fine roots).

- les racines fines $(5-20 \mathrm{~mm})$ des chênes rétablis ont une prospection en profondeur assez semblable (fig. 5) ; elles colonisent l'horizon induré. On note cependant, dans ce cas, que le sessile a un enracinement plus profond que le pédonculé. Le pédonculé non rétabli se distingue par une biomasse racinaire toujours plus faible que celle des chênes rétablis et ceci quelle que soit la profondeur. (On remarque notamment, dans ce cas, unc absence totale de racines fines à partir de $60 \mathrm{~cm}$ de profondeur).

Pour ce type de racines on n'observe donc pas de différence sensible entre les sessiles et pédonculés rétablis ; par contre, le pédonculé non rétabli cst caractérisé par une diminution de sa biomasse et une limitation de sa prospection en profondeur. 


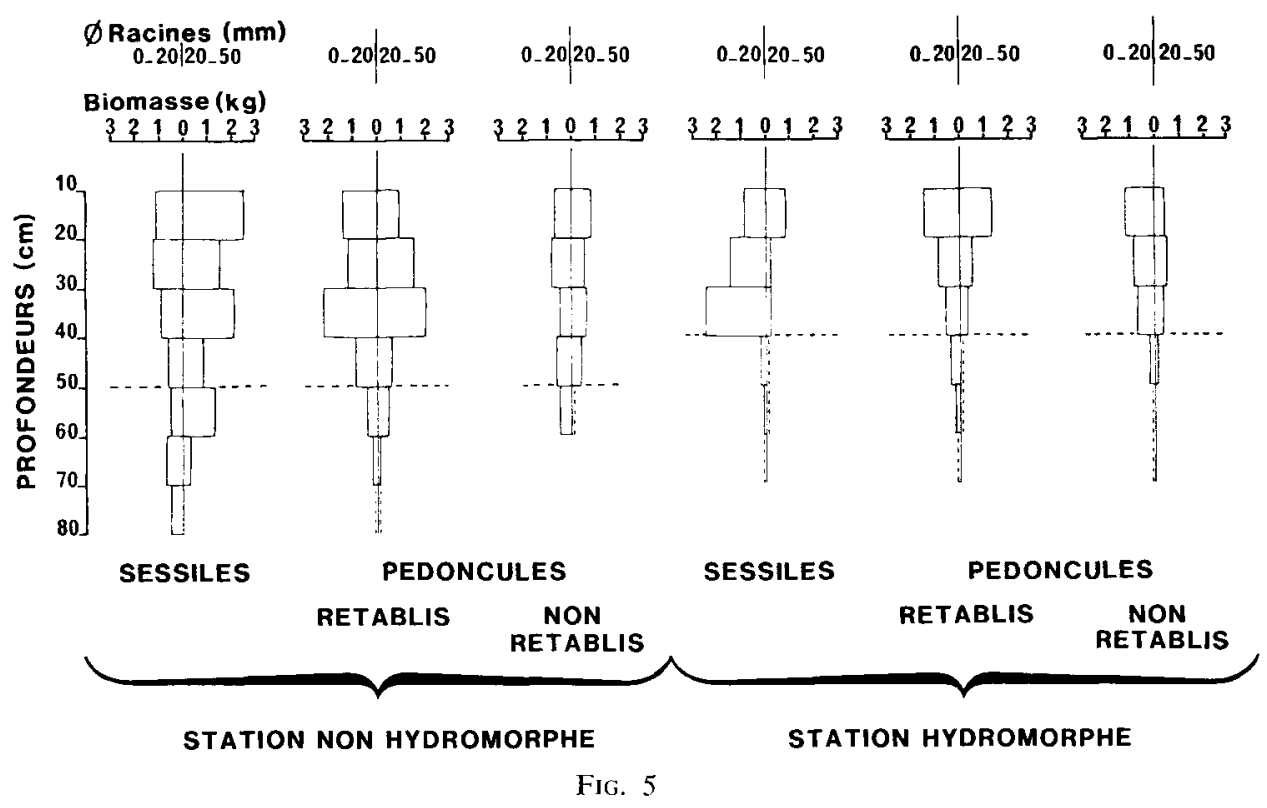

Répartition selon la profondeur des racines fines et movennes entre 1 et 2 mòtres de la souche (exprimée en $\mathrm{kg}$ de poids sec).

Biomass of fine and medium roots at different soil depths measured from 1 to 2 meters away from the stump ( $k g$ dry weight).

- pour les racines de diamètre compris entre 20 et $50 \mathrm{~mm}$ on arrive à des conclusions semblables :

- jusqu'à l'horizon induré, la prospection racinaire des chênes rétablis est semblable bien que l'induration semble être légèrement plus défavorable au pédonculé qu'au sessile;

- la prospection et la biomasse des grosses racines du pédonculé non rétabli sont moindres : le phénomène est très net dans les horizons profonds du sol mais on lc constate déjà dans les horizons supérieurs (entre 20 et $60 \mathrm{~cm}$ de profondeur, la biomasse racinaire est 2 à 3 fois plus petite que celle des chênes rétablis).

Cette étude, malgré ses limites, montre que sur les arbres rétablis, on n'observe pas de nette différence entre l'architecture racinaire du sessile et du pédonculé ; en revanche, il semble que le chêne sessile soit caractérisé par une prospection racinairc plus profonde notamment pour les très fines racines. Cette différence spécifique pourrait être à l'origine de la meilleure résistance du sessile à un stress hydrique, du moins sur station non hydromorphe.

Par contre, les pédonculés non rétablis se différencient nettement ; ils présentent un système racinaire plus superficiel et moins dense ; ces caractéristiques pourraient coïncider avec une plus forte sensibilité de ces arbres à un stress hydrique. 
Les observations effectuées dans la station hydromorphe $\left(\mathrm{H}^{+}+\mathrm{D}^{+}\right)$amènent à des conclusions assez semblables : la présence d'une nappe superficiclle limite en effet la progression racinaire aux 40 premiers centimètres du sol; il en résulte que les différences entre les 3 types d'arbres se trouvent estompées cependant ce sont toujours les pédonculés non rétablis qui présentent une biomasse plus faible. Mais sur cette station, la différence avec les chênes rétablis est moins prononcée que celle observée dans la station non hydromorphe.

\section{Les altérations pathologiques}

\subsection{Typologie des lésions}

Sur les souches et le départ des grosses racines, les lésions rencontrées peuvent être regroupées en quatre types principaux :

1) Les lésions sans mycélium apparent : il s'agit de plages nécrotiques brunes, fréquentes et généralement de faible surface.

Les trois autres types comportent du mycélium :

2) Le type A se caractérise par une écorce brune, morte mais cohérente (non fibreuse) et des palmettes blanches, continues, parcheminées, dont la morphologie évoque l'Armillaire. On peut distinguer :

a) un sous-type A 1 (assez rare) où la lésion est superficielle, de faible surface, et oì les palmettes sont présentes dans toute l'épaisseur de l'écorce :

b) un sous-type A 2 où la lésion est de grande taille et où les palmettes sont localisées au niveau du cambium et prennent une grande extension.

3) Le type $T$ est caractérisé par des taches corticales de couleur orangée à brune, qui sont à la fois fibreuses et suintantes. Le mycélium s'organise en petites palmettes discontinues comprises dans toute l'épaisseur de l'écorce (et pas spécialement au niveau du cambium). Comme pour le type $A$, on distingue deux sous-types qui semblent correspondre à deux étapes successives dans l'évolution de l'attaque :

a) dans le sous-type $T 1$, la lésion, de taille réduite, est le plus souvent limitée par un liseré noir. Elle n'atteint pas le cambium. Les palmettes sont peu visibles, les plages orangées fibreuses à mycélium alternent avec des plages brunes plus cohérentes ct sans mycélium ;

b) dans le sous-type $\mathrm{T} 2$, les taches, de plus grandes dimensions, sans liseré, humides, très décomposées, montrent un mycélium abondant. Ces taches atteignent le cambium et le dépassent : l'aubier sous-jacent peut être très décomposé.

4) Le type $X$ rassemble toutes les lésions comportant du mycélium et n'appartenant pas aux types A et T. Il s'agit toujours de nécroses de faible profondeur, n'attejgnant pas le cambium, souvent localisées dans les zones les moins vivantes du système racinaire (par exemple sur les sinus séparant les grosses racines).

Cette typologie a été, pour l'essentiel, retrouvée lorsqu'on a examiné les racines moyennes (de 5 à $20 \mathrm{~mm}$ de diamètre) des arbres dont on avait dégagé le système racinaire; Ic type $X$ n'était pas représenté dans cette catégorie de racines. Par contre, 
on a retrouvé les types $\mathrm{A}$ et $\mathrm{T}$. Par ailleurs, de nombreuses racines moyennes étaient nécrosées sans porter de mycélium visible.

\subsection{Importance quantilative des lésions et relation avec le dépérissement}

1) Etude des 197 arbres dont la partie superficielle du système racinaire a été cxaminéc.

La répartition de ces arbres selon leur lype et celui de la placette figure sur le tableau 3.

Les arbres ont en outre été répartis en quatre classes en fonction de la gravité des lésions fongiques sur la partie visible du système racinaire. Ces classes ont été délimitées de façon à rendre compte, à notre sens, du degré de perturbation que les dégâts entraînent pour l'arbre. Classe I (gravité nulle) : lésions très localisées de type $\mathrm{T} 1$ ou X, n'atteignant pas le cambium ; classe II (gravité faible) : présence de lésions de type T 2 (en plus de T1 et X), attcignant localement le cambium; classe III (gravité forte) : présence de lésions de type T 2 et A 1 dépassant le cambium et/ou recouvrant au moins 20 p. 100 de la surface ; classe IV (gravité très forte) : lésions de type T 2 et A 2 dépassant le cambium et recouvrant au moins 60 p. 100 de la surface.

\section{TABLEAU 3}

Répartition des effectifs des 197 arbres étudiés.

Distribution of the 197 oaks studied. Values are the number of trees found in each type of soil $\left(\mathrm{H}^{-}\right.$and $\left.\mathrm{H}^{+}\right)$and for each level of dieback $\left(\mathrm{D}^{-}\right.$and $\left.\mathrm{D}^{+}\right)$. All conditions as in table 1.

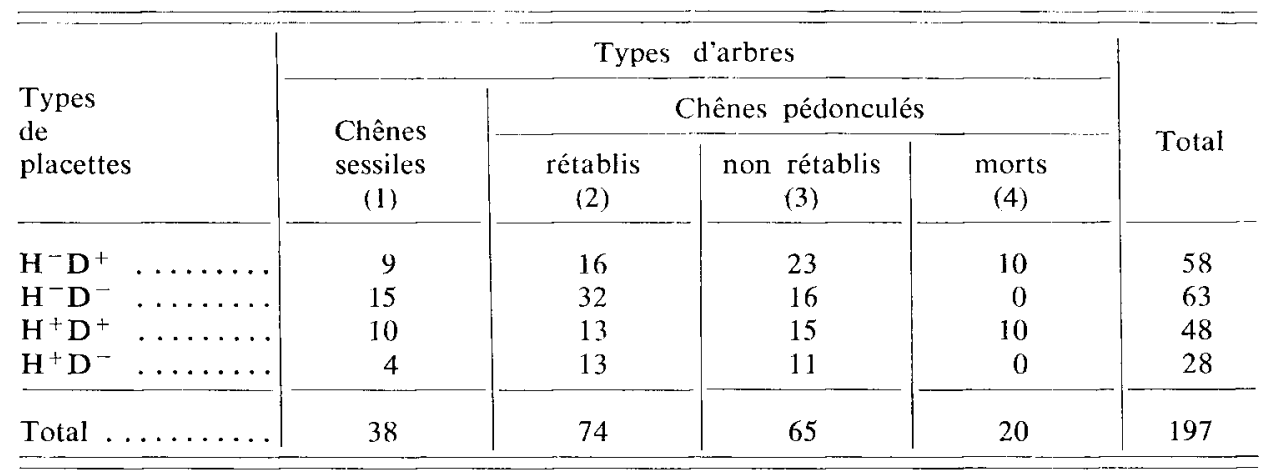

Le tableau 4 exprime la relation entre la vigueur des arbres et la gravité des attaques. Une première analyse de ces résultats permet de tirer les conclusions suivantes :

- Il existe une assez bonne corrélation entre le degré de dépérissement des arbres et le degré d'attaque par les champignons. Les chênes sessiles (1) sont significativement moins attaqués que les chênes pédonculés « rétablis " (2). (On a vu que le rétablissement était généralement plus tardif pour les pédonculés que pour les 


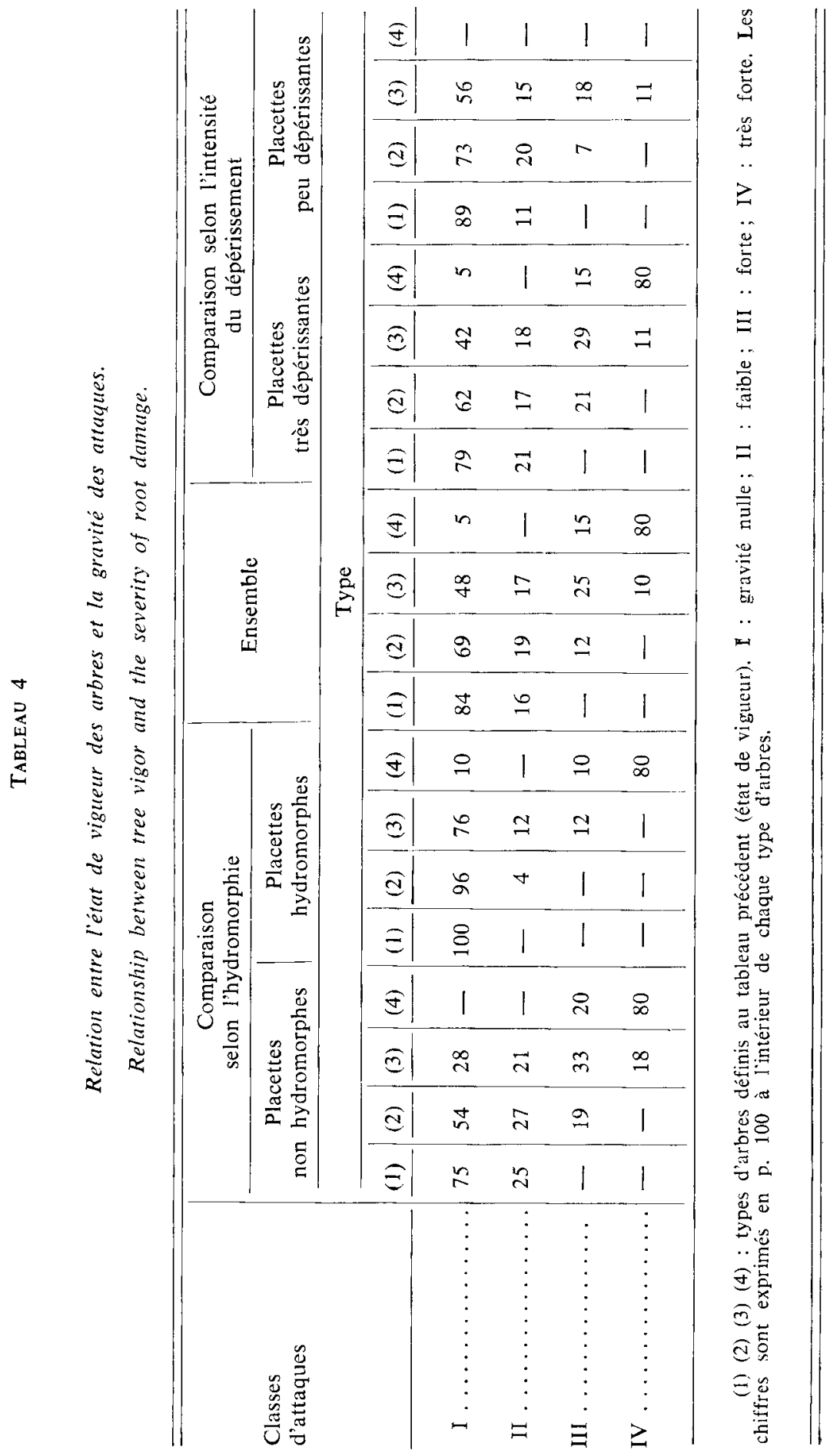


sessiles). Les pédonculés rétablis sont moins attaqués que les pédonculés non rétablis (3). Ces derniers sont moins attaqués que les pédonculés morts (4). (Ne figurent dans cette dernière catégorie que des arbres morts depuis moins de deux ans).

- Cette conclusion globale est surtout valable pour les sols non hydromorphes. En sols hydromorphes, les différences s'estompent (notamment entre chênes sessiles et chênes pédonculés rétablis). D’une façon générale, les infections fongiques sur arbes vivants sont nettement moins avancées sur sol hydromorphe.

- Pour une catégorie d'arbres donnée, les attaques fongiques sont plus importantes sur les parcelles très dépérissantes que sur les parcelles peu dépérissantes. Il est possible que la présence d'arbres morts et d'un plus grand nombre d'arbres dépérissants, en augmentant la quantité de bois mort disponible pour les champignons, provoque un accroissement du potenticl d'inoculum.

2) Etude des arbres dont les racines ont été dégagées.

Les résultats, brièvement résumés dans le tableau 5, concernent les sept arbres établis sur sol non hydromorphe (placette de type $H^{-} D^{t}$, parcelle 107). L'un de ces 7 arbres (le «pédonculé rétabli $\mathrm{n}^{\prime \prime} 3$ » du lableau 5) n'avait pas été pris en compte dans l'étude des caractéristiques de l'enracinement, car situé sur un sol plus profond.

a) Pour ce qui concerne les grosses racines, les résultats enregistrés sont en accord avec ceux qui ont été tirés de l'observation de la partie superficielle des systèmes racinaires. En-dessous de $40 \mathrm{~cm}$ de profondeur, on a retrouvé les mêmes types de lésions que dans les parties plus superficielles. La proportion de racines détruites semble indépendante de la profondeur, en revanche elle dépend de la distance à la souche, les racines étant d'autant plus attaquées qu'elles sont plus proches de la souche.

La proportion des tissus de la souche et des grosses racines détruits par les champignons était plus élevée pour les deux chênes pédonculés «non rétablis " que pour les autres arbres. L'un des deux montrait un système racinaire à peu près totalement décomposé par la pourriture fibreuse de type T 2.

Les chênes pédonculés rétablis se caractérisaient par l'hétérogénéité de la répartition des attaques fongiques sur les systèmes racinaires. L'un de ces 3 arbres était aussi faiblement attaqué que les 2 chênes sessiles.

b) Pour les racines moyennes (diamètre entre 5 et $20 \mathrm{~mm}$ ), on a noté une différence très importante entre les deux chênes sessiles, qui présentaient des taux de mortalité racinaire très faibles, et les cinq chênes pédonculés. Signalons aussi qu'à ce niveau. les lésions de type $A$ sont au moins aussi fréquentes que les lésions de type $T$, alors que ces dernières prédominent sur les grosses racines et la souche.

Les premiers dépouillements effectués pour les 6 arbres situés sur une parcelle à sol hydromorphe ( $\left.n^{\circ 1} 160\right)$ amènent à des conclusions assez différentes : l'impact global des champignons est bien moindre et on observe peu de différences entre le degré d'attaque des chênes sessiles et celui des pédonculés rétablis. 
PATHOLOGIE RACINAIRE DU CHÊNE

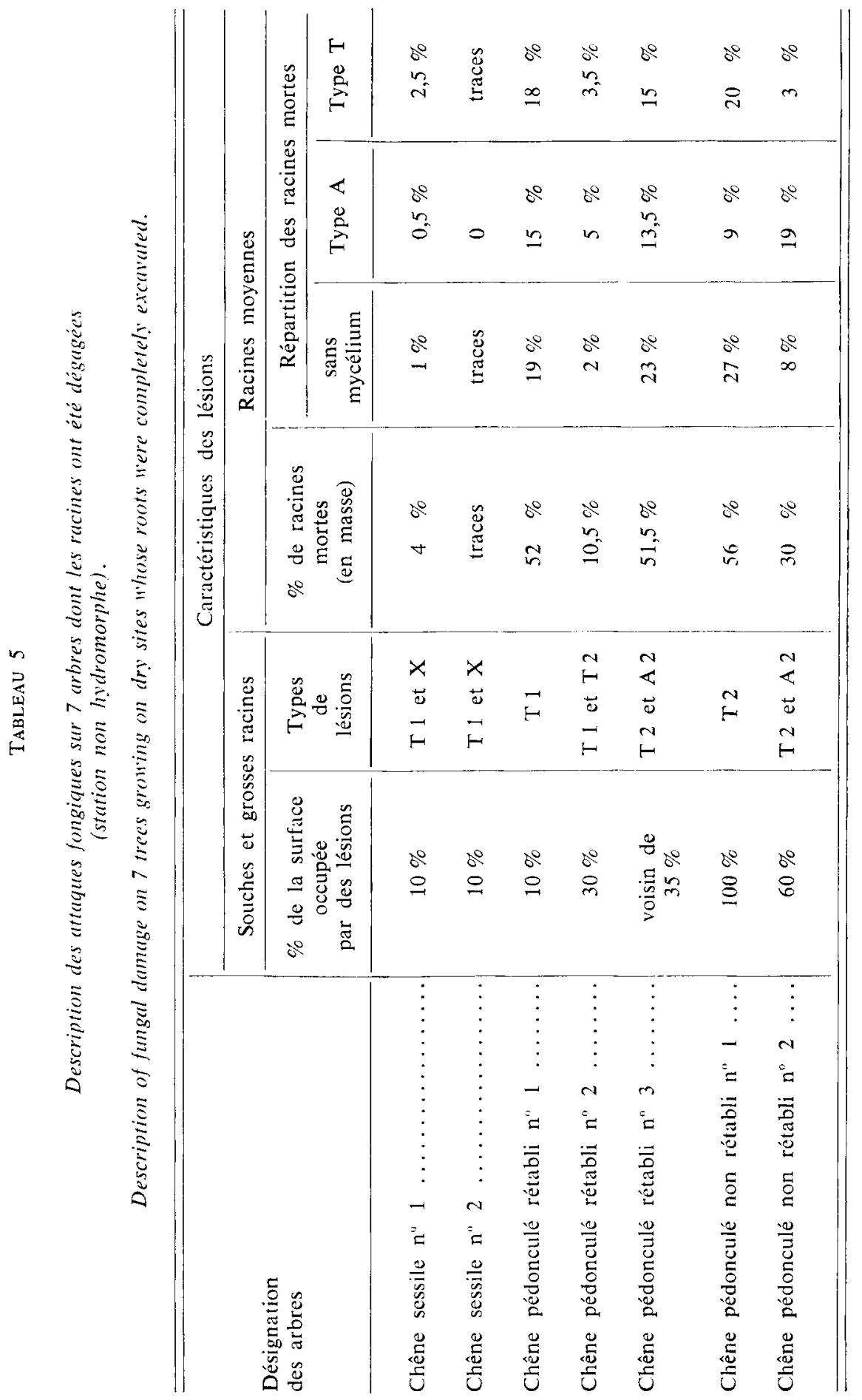




\subsection{Les champignons responsables}

Des isolements ont été effectués à partir des différents types de lésions. Nous avons tout particulièrement recherché les Basidiomycètes qui sont les agents classiques de dégradation du bois et dont le mycélium était reconnaissable dans la plupart des lésions :

- à partir des lésions de type A (aussi bien sur souches et grosses racines que sur racines moyennes), on a comme il était prévisible, isolé des Armillaires;

- à partir des lésions $T$, on a isolé, avec une fréquence élevée, l'Agaricale Collybia fusipes (Bull. ex Fr.) Quél.;

- à partir des lésions $X$, ont été isolécs plusieurs espèces de Basidionycètes, chacune avec de faibles fréquences. Ces espèces, dont certaines ont pu être identifiées, sont bien différentes de Collybia fusipes et semblent jouer un rôle assez marginal.

\subsubsection{Les Armillaires}

\subsubsection{Le problème systénatique}

On sait, depuis les travaux de Korhonen (1978), que ce champignon, qui était considéré comme une espèce unique et appelé, depuis Kummer et Quelet, Armillaria mellea, est en fait une espèce collective comportant, pour le continent européen, cinq espèces bien distinctes. Des travaux récents (Guillaumin \& Berthlicay, 1981 ; RishbeTH, 1982) ont montré que ces espèces diffèrent par de nombreux caractères (répartition géographique, écologic, pouvoir pathogène, etc.).

A Tronçais, nous avions déjà tenté d'identifier les espèces d'armillaires présentes (Guillaumin, non publié 1981) à partir des carpophores ou à partir des rhizomorphes souterrains, très abondants dans les sols de toute la forêt (en zones dépérissantes ct non dépérissantes). L'étude des carpophores nous avait permis d'identificr les 3 espèces Armillaria bulbosa (Barla) Romagn.= espèce «E» sensu Korhonen, Armillaria mellea (Vahl) Kummer sensu stricto = espèce «D»Korhonen, et enfin l'espèce «B » de Korhonen, cette dernière semblant très rare. Quant aux isolements effectués à partir des rhizomorphes, ils avaient toujours livré exclusivement $A$. bulbosat.

Il était done intéressant de déterminer les espèces d'armillaires impliquées dans les lésions du système racinaire des chênes. Les méthodes d'identification utilisées à cette fin ont élé décrites dans une publication déjà citée (Gurllaumin \& Berthelay, 1981).

\subsubsection{Résullats des déterminations}

- Echantillons provenant des souches et des grosses racines :

Sur 13 isolats ayant fait l'objet d'une détermination, 10 appartenaient à l'espèce A. bulbosa et 3 à $A$. mellea sensu stricto.

Ces résultats pcuvent êtrc complétés par ceux obtenus à partir d'échantillons du même type et provenant des chênaies dépérissantes du Pays Basque et du Béarn : sur 17 isolats identifiés, 10 appartenaient à $A$. bulbosa et 7 à $A$. mellea. 
- Echantillons provenant de racines moyennes :

Sur 17 isolements d'Armillaire réussis, nous n'avions malheureusement conservé et identifié que trois souches. Elles appartenaient toutes trois à $A$. mellea sensu stricto.

\subsubsection{Collybia fusipes}

Faute de disposer de la phase sexuée permettant seule une identification précise, nous avons dû, pendant plus d'un an, désigner les isolats de cette espèce par un nom de code. Ce n'est qu'à partir de mars 1983 qu'ont pu être obtenus, en culture pure, des carpophores parvenus à complète maturité. Ces carpophores ont été déterminés comme pouvant appartenir à l'espèce Collybia fusipes.

Nous avons tenté de confirmer cette identification en effectuant des isolements à partir de lésions racinaires porteuses de carpophores de Collybia fusipes de diverses provenances (Meurthe-et-Moselle, Puy-de-Dôme, Roumanie), pendant l'été 1983. Les isolats obtenus présentaient, en culture pure, la même morphologie macro et microscopique que nos isolats de T 1-4. Une culture de Collybia fusipes obtenue à la Mycothèque de Baarn (Pays-Bas) s'est également avérée en tous points semblable à nos isolats (Delatour \& Guillaumin, 1984).

Les isolats de cette espèce en culture présentent une morphologic assez originale, caractérisée entre autres par l'apparition précoce d'une croûte centrale brun-roux, qui se recouvre ensuite de gouttelettes sombres, par la sécrétion dans le milieu d'un pigment roux, et enfin par l'apparition tardive de "lignes noires " circulaires constituées d'articles mycéliens isodiamétriques et mélanisés.

Le mycélium présente des boucles à toutes les cloisons. Du point de vuc enzymatique, la laccase et la peroxydase sont présentes, la tyrosinase est absente. L'espèce possède, in vitro, un pouvoir lignivore élevé.

Les basidiospores sont de petite taille $(6-7, \mathrm{t})$, hyalines et dépourvues d'ornementations.

A Tronçais, en sols non hydromorphes, la Collybie apparaît être l'agent fongique de dégradation des chênes vivants le plus fréquent et le plus dommagcable, au moins au niveau des souches et des grosses racines. Les attaques jeunes du champignon (type « $T 1 》)$ sont très nombreuses et faciles à mettre en évidence, ce qui n'est pas le cas des attaques jeunes d'Armillaire.

Ces lésions « T 1 » se rencontrent notamment sur des arbres d'apparence saine, pédonculés rétablis ou même sessiles. Elles sont alors, très fréquemment, entourées d'un liseré brun qui correspond à un liège réactionnel élaboré par une assise génératrice subéro-phellodermique. L'activité de cette assise est apparcmment efficace, du moins sur les arbres sains : à l'intérieur de la lésion, les parenchymes corticaux sont totalement décomposés, seuls subsistent des faisceaux de fibres sclérenchymateuses. Mais le champignon semble ne pas franchir aisément la barrière lićgeuse : de telles lésions peuvent être considérées comme «latentes»: le champignon reste vivant, mais est incapable de s'étendre sur ces arbres restés ou redevenus vigoureux.

Sur les chênes pédonculés non rétablis, les lésions latentes T 1 se transforment en lésions évolutives de type $\mathrm{T} 2$, tous les intermédiaires pouvant être rencontrés. 
Il est à noter que ce champignon a également été mis en évidence sur certains arbres dépérissants des chênaies basco-béarnaises, ainsi qu'en Lorraine.

\section{3.j. Les autres espèces}

Quinze autres cspèces de Basidiomycètes ont été isolées. Ces isolements provenaient, le plus souvent, de lésions de type $X$, plus rarement de lésions de type $T$; dans ce second cas, ce résultat peut correspondre soit à une typologie insuffisamment précise, soit à une surinfection de lésions « $\mathrm{T} 2$ » par d'autres Basidiomycètes.

Sur les quinze espèces, trois ont pu être déterminées; il s'agit de trois Agaricales :

- Collybia platyphylla Fr. ex Pers. était aisément reconnaissable aux rhizomorphes cylindriques et blanes, attachés à la fois aux lésions et à la base des carpophores ;

- Marasmias bresadolae Kühn et Romagn. et Mycena praecox Vel. ont fructifie sur des blocs de bois infectés artificiellement, puis laissés à-demi enfouis dans de la tourbe, en scrre, en conditions non stériles. Ces deux dernières espèces ont été déterminées par D. Lamoure (Université de Lyon I).

En tout état de cause, Ic rôle pathogène de ces Basidiomycètes « divers » apparaît extrềnement limité.

\section{Conclusions et discussion}

Contrairement à la plupart des travaux antérieurs sur ce sujet et grâce à une collaboration pluridisciplinaire, notre étude a pu s’insérer dans un cadre écologique précis. De plus, elle a pu se fonder sur une appréciation objective du dépérissement, fournic par la mesure des accroissements annuels. Enfin, les observations superficielles des racines ont pu être complétées par l'investigation presque complète de quelques arbres, ce qui avait rarement été le cas dans les études antéricures.

Il scmble que les différences de comportement entre le chêne sessile ct le chêne pédonculé ne puissent être expliquées ni par la structure ct la profondeur de leur système racinaire, ni par des différences dans les biomasses racinaires.

Par contre, ces différences apparaissent nettement entre les chénes pédonculés rétablis et non rétablis. Mais elles peuvent être dues simplement à la destruction par les champignons d'ume partic importante du système racinaire sur les arbres non rétablis.

En effet, en stations non hydromorphes, il est apparu une relation assez étroite entre l'état de dépérissement des arbres et leur degré d'infection par les champignons des racines. Cette constatation n'est pas suffisante à elle seule pour affirmer que les champignons des racines contribuent au dépérissement. En effet, il se pose, comme chaque fois qu'il est question de parasitisme de faiblesse, le «problème des causes et des conséquences » : l'affaiblissement (d'origine écologique) des arbres a-t-il contribué au développement des champignons? Et à l'inverse, ce développement a-t-il contribué à accentuer l'affaiblissement des arbres, jusqu'à le rendre irréversible? 
S'il est facile de répondre par l'affirmative à la première question, il est plus délicat d'apporter une réponse solidement étayée à la seconde : les infections fongiques contribuent-elles au dépérissement des chênes pédonculés et, en particulier, sont-elles bien le facteur qui a fait basculer dans le mauvais sens des arbres affaiblis qui se trouvaient «à la croisée des chemins» autour de l'année 1978? Pour pouvoir répondre avec certitude, il aurait fallu commencer l'étude à ce moment-là et démontrer ensuite que ce sont les arbres les plus atteints par les champignons en 1978 qui ont continué à décliner pendant que les autres se rétablissaient. En l'absence de cette preuve directe, nous ne pouvons que faire appel à des arguments indirects :

- d'une part, bien que ce ne soit pas le cas général, on trouve parfois des infections évolutives sur des arbres vigoureux : done l'implantation des champignons, et même le passage de l'état latent à l'état évolutif peuvent précéder le dépérissement, ils n'en sont pas toujours une simple conséquence;

- d'autre part, certains chênes pédonculés non rétablis, mais encorc bien vivants, présentent des taux d'infection de leur système racinaire très élevés, parfois proches de 100 p. 100. Le pronostic de ces arbres est facile à établir : le simple bon sens indique que, passé un certain seuil d'envahissement, le rétablissement devient impossible.

Cette corrélation entre stade de dépérissement et degré d'infection semble ne plus être valable sur les sols hydromorphes. On peut, à ce sujet, formuler deux hypothèses, qui sont complémentaires : d'une part, il est tout à fait plausible que la présence d'une nappe proche de la surface pendant six mois de l'année crée des conditions défavorables au développement des champignons, la Collybie en particulier. Mais d'autre part, la présence de cette nappe peut également porter le «coup de grâce " aux arbres affaiblis, remplaçant dans ce rôle les champignons. En sols hydromorphes, on aurait donc un système dans lequel la sécheresse serait responsable de l'affaiblissement des chênes pédonculés, puis l'excès d'eau de leur mort, les champignons ne jouant dans ce cas qu'un rôle secondaire.

L'un des résultats inattendus de notre étude est que l'Armillaire (ou les Armillaires) ne sont pas les seuls champignons intervenant dans le processus; en sols non hydromorphes, elles sont même moins fréquemment rencontrées que Collybia fusipes.

Il peut paraître surprenant que les auteurs anciens n'aient jamais signalé cette espèce pathogène, qui n'est pas localisée à la région de Tronçais puisque nous l'avons retrouvée, jouant le même rôle, dans les chênaies basco-béarnaises. Peut-être, en l'absence d'isolements, les palmettes abondantes présentes dans les lésions «T 2 " avaient-elles été confondues avec des palmettes d'armillaire (nous avons nous-mêmes commis cette erreur au tout début de notre étude). Il faut signaler également que l'adjonction de benzimidazoles dans les milieux d'isolement des Basidiomycètes (qui n'a été adoptée que récemment par les mycologues) a bien amélioré l'efficacité de ces isolements.

Selon certaines interprétations, le développement de la Collybie pourrait être un phénomène récent, et le dépérissement du chêne pédonculé serait alors, au moins partiellement, lié à ce développement et apparaîtrait donc comme étant, en partie, de nature épidémique. Nous ne sotscrivons pas à cette interprétation : il s'agit visiblement d'un parasite d'équilibre, espèce saprophyte banale de l'écosystème et dont le pouvoir pathogène n'a été révélé que grâce à des conditions écologiques et climatiques très 
particulières. La Collybie est présente sur les arbres vigoureux sous forme de lésions latentes susceptibles de se transformer en lésions évolutives sur les arbres affaiblis. Nous avons pu constater son abondance sur les vicilles souches, dont clle détruit l'écorce et l'aubier, ne laissant subsister que le duramen.

Bien entendu, dans l'état actuel des investigations, on ne peut pas affirmer que le champignon est incapable de se comporter également comme un parasite primaire, susceptible d'évoluer sur des arbres n’ayant subi aucun affaiblissement. Seules des inoculations artificielles pourront peut-être apporter la réponse à cette question.

Le cycle infectieux de cette espèce reste à étudier. Toutefois, Ie grand nombre des lésions latentes observées sur certains systèmes racinaires laisse penser que le mycélium pourait être présent dans le sol lui-même, et pas seulement dans le bois mort contenu dans le sol, comme c'est le cas pour les armillaires.

Une autre question que l'on peut se poser est la raison pour laquelle le chêne pédonculé est plus attaqué par les champignons (en particulier la Collybia) que le chêne sessile. Est-ce seulement parce qu'il est plus affaibli par l'ensemble des facteurs ćcologiques? Ou existe-t-il en outre une sensibilité intrinsèque plus élevée du pédonculé, qui se manifesterait mêne sur des arbres non stressés? Là encore, des inoculations artificielles pourraient permettre de trancher.

Enfin, notre étude nous a suggéré une certaine «répartition des rôles » entre les 3 espèces de champignons impliquées dans le dépérissement du chêne pédonculé : Collybia fusipes, Amillaria mellea sensu stricto et Armillaria bulbosa. A. mellea a pu être isolé sur racines moyennes, loin du tronc, ce qui n'a pas été le cas pour' bulbosa, qui semble, en revanche, plus fréquent sur les souches, surtout aux stades avancés du dépérissement.

Il semble donc que la Collybie et $A$. mellea interviennent plus précocement que A. bulbosa, c'est-à-dire sur des arbres moins affaiblis : Collybia domine, de loin, sur les souches et les grosses racines. C'est à un stade plus avancé que $A$. bulbosa, qui est toujours présent à l'extéricur des souches par ses rhizomorphes, deviendrait capable de pénétrer au niveau du collet, conculrençant ou même supplantant la Collybie. Cette armillaire pett notamment envahir rapidement, par progression dans le cambium, le trone des arbres morts ou mourants, ce que ne peut faire la Collybie.

Cette différence de rôle entre Armillaria mellea et $A$. bulbosa serait en accord avec les publications récentes, qui attribuent à mellea une agressivité supérieure à celle de bulbosa (Guillaumin \& Berthelay, Rishbeth, articles cilés). Ces conceptions restent toutefois fragiles et ont besoin d'être étayées par des observations et des isolements en plus grand nombre, et portant sur des zones géographiques plus variées.

\section{Remerciements}

Le présent travail a nécessité le recours à l'aide technique de nombreuses personnes que nous tenons à remercier particulièrement : MM. M. Adrian, P. Bonnaud, C. BRechet, Y. Lefevre, J.L. Renoux, M. Thomas, ainsi que de la compréhension active de l'O.N.F., Centre de Moulins et de son Service local en forêt de Tronçais. Il a également bénéficié de l'aide financière de 1'A.T.P.-I.N.R.A. «Dépérissement du chêne » $\left(\mathrm{n}^{\circ} 4265\right)$. 


\author{
Summary \\ Root pathology of Quercus robur $L$. \\ dieback in the Tronçais Forest (of Central France)
}

Since 1978, dicback of 70 to 120 years old oaks has been observed mainly in two regions of France; the plains of Central France and the «Pays Basque ». The etiology of the phenomenon is complex, pendunculate Oak (Quercus robur L.) is the only species affected, since the other oaks $(Q$. petraea Sm. and $Q$. borealis Michx.) growing in the same stands are not affected.

The onset of the dieback is related to climatic and edaphic variables. It was induced by the 1976 drought, but only pedunculate oaks were affected where root development was hindered by a stone bed or a high water table.

This study was done in the Tronçais Forest of Central France and examined the relationship between higher fungi which attacked $Q$. robur roots and the dieback phenomenon. Several methods of examining the roots were used, including excavation of the entire root system. The degree of root infection was correlated with the development of the trec (radial growth pattern determined by dendrochronology).

It appeared that root rot fungi play an important role in the dicback phenomenon. They may not interfere during the initial stages of decline. However, their presence can explain the death of some trees since 1978 while non-infected, neighbouring trees recovered during this period.

Three species of basidiomycetes were involved in the decline of $Q$. robur:

1) Two species of \& honcy fungi $» ;$ A millaria mellea (Vahl) Kummer sensu stricto and Armillaria bubosa (Barla) Romagn. which are prevalent in wet soils. A. mellea which attacks roots earlier than $A$. bulbosa, can affect roots of mid-diameter and can do so at some distance from the trunk. In contrast $A$. bulbosa invades the base of dying trees.

2) A marasmioid species, which was later identified as Collybia fusipes (Bull. ex Fr.) Quél. by obtaining fruiting bodies in pure culture. This fungus is the most prevalent pathogen in dry soils. It is frequently found on healthy trees (including $Q$. petraea) in its latent form and not as growing lesions. Once the trees are weakened by an environmental stress, the lesions develop and the fungus can invade the entire root system causing a wet, orange rot in the bark and sapwood. This is the first time this species has been recognized as a pathogen.

\title{
Références bibliographiques
}

BERnaRd C., 1982. Contribution à l'éude du dépérissement du chêne en forêt de Tronçais. Intervention des champignons pathogènes des racines (I.N.R.A. - Clermont-Ferrand). Mémoire de $3^{\circ}$ année E.N.I.T.E.F., 101 pages.

Becker M., Livvy G., 1982. Le dépérissement du chêne en forêt de Tronçais. Les causes écologiques. Ann. Sci. For., 39 (4), 439-444.

Beckfr M., Levy G., 1983. Le dépérissement du chêne. Les causes écologiques (exemple de la forêt de Tronçais) et premières conclusions. Rev. For. Fr., XXXX (5).

BuFfet M., 1983. Le dépérissement du chêne en forêt soumise. Rev. For. Fr., XXXV (3), 199-204.

Delatour C., 1983. Les dépérissements de chênes en Europe. Rev. For. Fr., XXXV (3), $265-282$.

Delatour C., Guillaumin J.J., 1984. Un pourridié méconnu : le Collybia fusipes (Bull. ex Fr.) Quél. C.R. Acad. Agr. Fr., 70 (1), 123-126.

Guillaumin J.J., Berthelay Suzanne, 1981. Détermination spécifique des Armillaires par la méthode des groupes de compatibilité sexuclle. Spécialisation écologique des espèces françaises. Agronomie, 1 (10), 897-908.

KORHONEN K., 1978. Interfertility and clonal size in the Armillariella mellea complex. Karstenia, 18, 31-42.

Riom J., 1981. Le dépérissement du chêne en forêt de Tronçais. Etude par photographies aériennes. Document interne, I.N.R.A. Télédétection.

Rishbetri J., 1982. Species of Armillaria in southern England. Plant Pathol, 31, 9-17. 